\title{
Clinical outcomes following trifocal diffractive intraocular lens implantation for age-related cataract in China
}

This article was published in the following Dove Press journal:

Clinical Ophthalmology

Yang Yang

Huibin Lv

Yuexin Wang

Xiaodan Jiang

Mingzhou Zhang

Xuemin Li

Department of Ophthalmology, Peking University Third Hospital, Beijing, China
Correspondence: Xuemin $\mathrm{Li}$ Department of Ophthalmology, Peking University Third Hospital, 49 North Garden Road, Haidian District, Beijing 10019I, China

$\mathrm{Tel}+86$ I39 II 254862

Email I39II254862@I63.com
Purpose: The aim of this study was to evaluate visual, refractive and patient satisfaction outcomes following implantation of a trifocal diffractive intraocular lens (IOL).

Patients and methods: This prospective, consecutive study included patients undergoing lens phacoemulsification of cataract and implantation of a trifocal diffractive IOL (AT LISA tri 839MP). Visual outcomes, including near, intermediate and distance visual acuity (VA), refractive error, contrast sensitivity, defocus curve and patient satisfaction were assessed preoperatively and postoperatively.

Results: Thirty IOLs were implanted in 26 patients. Distance VA improved significantly from $0.70 \pm 0.45$ to $0.08 \pm 0.11 \operatorname{logMAR}(p<0.0001) 1$ week postoperatively, and to $0.07 \pm 0.13 \log$ MAR $(p<0.0001)$ at 1 month and $0.05 \pm 0.10 \operatorname{logMAR}(p<0.0001)$ at 3 months. Uncorrected near and intermediate VA, as well as corrected near, intermediate and distance VA, were stable and maintained during the follow-up period. Preoperative anterior chamber depth demonstrated an association with effective adjustment of postoperative spherical equivalent using a regression formula ( $p=0.007)$. No significant differences were observed for VA at defocus curves of 0 to $-3 \mathrm{D}$. Contrast sensitivity at each spatial frequency improved significantly at 1 week, 1 month and 3 months under photopic and photopic with glare conditions. Under mesopic and mesopic with glare conditions, significant differences were observed postoperatively at low and medium spatial frequencies. Patients reported a high level of satisfaction and an absence of glare or halo 3 months postoperatively.

Conclusion: In this study, the trifocal diffractive IOL provided excellent visual performance at all distances and improved contrast sensitivity under different conditions, resulting in high levels of patient satisfaction and spectacle independence in Chinese patients.

Keywords: visual acuity, patient satisfaction, trifocal diffractive intraocular lens, spherical equivalent, contrast sensitivity defocus curves

\section{Introduction}

Cataract is a widespread ocular disorder characterized by progressively impaired visual acuity (VA) caused by lens opacity, and ranked as the most prevalent, blindnesscausing, ocular disease by the World Health Organization. ${ }^{1,2}$ As the use of phacoemulsification becomes more common, and instrumentation, techniques and improvements to intraocular lens (IOL) design continue to evolve, the postoperative goal of visual restoration has shifted to also improve the predictability of visual outcomes and to improve patient satisfaction and quality of life. ${ }^{3,4}$ Traditional IOLs offer a particular field of focus, often allowing for clear vision only at near or distance, but not both. Currently, multifocal IOLs deliver the capability to support clear VA at distance, 
intermediate and near..$^{5-7}$ There are four main categories of multifocal IOLs currently available, including refractive, diffractive, refractive-diffractive and accommodating. ${ }^{8}$ A new diffractive IOL with a trifocal design is the AT LISA tri 839MP (Carl Zeiss Meditec AG). ${ }^{9,10}$ Previous studies have demonstrated that the AT LISA tri 839MP provides sustainable restoration of VA after cataract surgery for near, intermediate and distance. ${ }^{1-13}$ Achieving the ideal, desired postoperative VA depends on precise ocular measurement, lens calculation and proficiency of surgical skill. Modern IOL calculation formulas tend to demonstrate similarly accurate refractive outcomes in eyes with axial lengths (ALs) within a normal range; however, formula accuracy differs in eyes with shorter or longer ALs. The Haigis formula incorporates the AL into the calculation; however, it is noted in clinical practice that small errors in postoperative refractive spherical equivalent still exist. ${ }^{14,15}$

The aim of this study was to evaluate the visual, refractive and patient satisfaction outcomes following implantation of a trifocal diffractive IOL and further investigate the adjusted formula in order to eliminate the difference in refractive error between postoperative and preoperative calculations.

\section{Patients and methods}

Thirty eyes of 26 patients undergoing lens phacoemulsification of cataract and implantation of a trifocal diffractive IOL (AT LISA tri 839MP; Carl Zeiss Meditec AG) for age-related cataract were enrolled in this prospective, consecutive case study. The study was conducted in accordance with the ethical principles based on the Declaration of Helsinki and its amendments, consistent with Good Clinical Practices and local regulatory requirements. Written informed consent was obtained from all study subjects, and the protocols were reviewed and approved by the institutional review board at the Peking University Third Hospital, where the study was conducted.

Patients were excluded from the study if they had corneal astigmatism $>1.0 \mathrm{D}$, other refractive media opacity, corneal disease, pupillary diseases, glaucoma, high myopia, macular degeneration, optic neuropathy, uveitis, retinal detachment, diabetic retinopathy, hypertensive retinopathy or complicated systemic disease or if they were incapable of completing the study-related visits.

Preoperative assessments included a complete ophthalmologic examination, comprising an evaluation of uncorrected distance VA (UDVA) (4 m, Early Treatment of Diabetic Retinopathy Study [ETDRS]), Goldmann applanation tonometry, slit-lamp ophthalmoscopy, biometry
(IOL Master version 4.3; Carl Zeiss Meditec AG) and contrast sensitivity under photopic, photopic with glare, mesopic and mesopic with glare condition at 1.5, 3, 6, 12 and $18 \mathrm{~cd} / \mathrm{m}^{2}$ (CSV-1000; VectorVision).

Postoperative examinations were conducted at 1 week, 1 month and 3 months following cataract extraction and IOL implantation. Postoperative assessments were identical to preoperative examinations, with the addition of monocular corrected distance VA (CDVA; 4 m, ETDRS), monocular uncorrected intermediate VA (UIVA) and corrected intermediate VA (CIVA; 66 m, modified ETDRS for Europeanwide use for near- and intermediate-distance recordings; Precision Vision), monocular uncorrected near VA (UNVA) and corrected near VA (CNVA; $33 \mathrm{~cm}$, modified ETDRS for European-wide use for near- and intermediate-distance recordings; Precision Vision). At 3 months postoperatively, patients underwent a defocus curve assessment and completed a satisfaction questionnaire.

\section{Defocus curve}

Defocus curves for monocular vision were obtained by applying ETDRS charts at $4 \mathrm{~m}$. The measurements were performed using distance correction, and VA was recorded according to varying defocus values (from +2.0 to $-5.0 \mathrm{D}$ in $0.5 \mathrm{D}$ steps) and represented in a two-dimensional graphic (spherical blur in the $\mathrm{x}$-axis and VA in the y-axis).

\section{Satisfaction questionnaire}

The satisfaction questionnaire was a self-developed questionnaire including questions about the patients' level of satisfaction with surgical outcome, degree of spectacle independence and difficulty performing vision-related activities after 3 months. Specifically, patients were asked about performing the following activities (grading: $1=$ Never, $2=$ Sometimes, or 3=Always): experience discomfort when performing near-vision tasks; when reading, experience headache for $>15$ minutes; when reading, experience clarity of text only for a period of time, and then text becomes unclear to them and cannot be refocused for a period of time; need for spectacle correction (at near, distance or both); lack of clear vision at distance; inability to engage in computer work for prolonged periods; vision difficulties when driving; and presence of glare or halos at night.

\section{Surgical technique}

All surgeries were performed by an experienced ophthalmologist using a standard technique of coaxial incision phacoemulsification for cataract extraction. Following routine 
disinfection, topical anesthesia and mydriatic drops were instilled in all cases and the surgical area was exposed using a speculum. The main incision was made into the limbus at the 10 o'clock position and a viscoelastic agent was injected. The lens nucleus and cortex were hydrodissected with balanced salt solution. Phacoemulsification-assisted incision was made at the 2 o'clock position and a $5 \mathrm{~mm}$ circular capsulorhexis was performed. After phacoemulsification, a two-tube aspiration needle was used to remove the residual cortex. BLUEMIXS 180 injector (Carl Zeiss Meditec AG) with AT LISA tri 839MP triple focus IOL (constant 118.8) was implanted through a $1.6 \mathrm{~mm}$ incision into the capsule. The IOL power was predicted preoperatively using the Haigis formula.

\section{Statistical analysis}

Statistical analysis was performed using SPSS (version 22.0; International Business Machines Corp.). Data with a normal linear distribution were represented by mean \pm standard deviation. For parametric statistical analysis, repeated measured analysis of variance was applied to assess the significance of differences between preoperative data and postoperative data at 1 week, 1 month and 3 months, whereas the Wilcoxon test was applied for nonparametric measurements. Correlation coefficients (Pearson or Spearman depending on whether normality could be assumed) were used to assess the correlation between preoperative variables and postoperative spherical equivalent at 3 months. Linear and nonlinear regression analyses were conducted to make conclusions regarding the adjusted formula. $p<0.05$ was considered statistically significant.

\section{Results}

Thirty eyes of 27 patients, of whom 13 were males, were included in the study and all patients completed the 3-month follow-up visit. No complications related to the procedure such as posterior capsule rupture, endophthalmitis or corneal decompensation were observed. The mean age of the patients at enrollment was $65.4 \pm 7.8$ years (53-81 years). Preoperative patient parameters are summarized in Table 1 . The mean preoperative $\mathrm{AL}$ was $23.55 \pm 0.82 \mathrm{~mm}$. The mean preoperative anterior chamber depth (ACD) was $3.01 \pm 0.48 \mathrm{~mm}$ and the mean preoperative estimation error for Haigis was $0.02 \pm 0.13$.

\section{VA and refraction}

Visual and refractive outcomes preoperatively and at each time point during the follow-up period are summarized
Table I Preoperative refractive and biometric characteristics

\begin{tabular}{lll}
\hline Characteristics & Mean \pm SD & Range \\
\hline Age (years) & $65.36 \pm 7.78$ & $53-81$ \\
Axial length $(\mathrm{mm})$ & $23.55 \pm 0.82$ & $22.32-26.63$ \\
Anterior chamber depth $(\mathrm{mm})$ & $3.01 \pm 0.48$ & $2.37-4.08$ \\
Flattest meridian $(\mathrm{K} 1)$ & $43.31 \pm 1.40$ & $40.19-46.63$ \\
Steepest meridian $(\mathrm{K} 2)$ & $43.90 \pm 1.35$ & $41.62-46.75$ \\
Preoperative estimation error & $0.02 \pm 0.13$ & $0.15-4$ \\
\hline
\end{tabular}

Abbreviation: SD, standard deviation.

in Table 2. UDVA was significantly better at 1 week $(p<0.0001), 1$ month $(p<0.0001)$ and 3 months $(p<0.0001)$ postoperatively, as compared to the baseline. All of the visual parameters including UDVA, UIVA, UNVA, CDVA, CIVA and CNVA were stable during the follow-up period. The proportion of eyes that achieved UDVA, UIVA and UNVA of $0.3 \log$ MAR or better at 3 months postoperatively was $100 \%$, $83.3 \%$ and $87.5 \%$, respectively. Similarly, $100 \%, 91.7 \%$ and 91.7\% of eyes achieved CDVA, CIVA and CNVA of 0.30 $\log$ MAR or better, respectively.

No significant difference was observed in refractive cylinder diopter during any of the follow-up visits, when compared with preoperative corneal astigmatism (1 week, $p=0.659$; 1 month, $p=0.965 ; 3$ months, $p=0.427$ ). At month 3 , mean spherical equivalent was $-0.05 \pm 0.65 \mathrm{D}$, sphere was $0.14 \pm 0.70 \mathrm{D}$ and cylinder diopter was $-0.50 \pm 0.39 \mathrm{D}$. There was no significant difference in sphere, cylinder or spherical equivalent at any of the postoperative time points.

\section{Adjusted formula}

Further analysis was undertaken to compare the correlation between preoperative variables including $\mathrm{ACD}, \mathrm{AL}$,

Table 2 Visual acuity and refraction preoperatively and I week, I month and 3 months after surgery

\begin{tabular}{lllll}
\hline & \multirow{2}{*}{ Preoperative } & \multicolumn{3}{l}{ Postoperative } \\
\cline { 3 - 5 } & & I week & I month & 3 months \\
\hline Astigmatism (D) & $-0.58 \pm 0.29$ & - & - & - \\
Sphere (D) & - & $0.11 \pm 0.45$ & $0.35 \pm 0.79$ & $0.14 \pm 0.70$ \\
Cylinder (D) & - & $-0.45 \pm 0.5 I$ & $-0.63 \pm 0.44$ & $-0.50 \pm 0.39$ \\
SE (D) & - & $-0.09 \pm 0.41$ & $0.05 \pm 0.67$ & $-0.05 \pm 0.65$ \\
UDVA & $0.70 \pm 0.45$ & $0.08 \pm 0.11$ & $0.07 \pm 0.13$ & $0.05 \pm 0.10^{*}$ \\
UIVA & - & $0.22 \pm 0.11$ & $0.23 \pm 0.13$ & $0.23 \pm 0.12$ \\
UNVA & - & $0.23 \pm 0.15$ & $0.19 \pm 0.14$ & $0.21 \pm 0.15$ \\
CDVA & - & $0.03 \pm 0.09$ & $0.02 \pm 0.11$ & $-0.01 \pm 0.08$ \\
CIVA & - & $0.18 \pm 0.14$ & $0.19 \pm 0.12$ & $0.18 \pm 0.13$ \\
\hline
\end{tabular}

Note: ${ }^{*} p<0.05$ vs I week postoperatively.

Abbreviations: SE, spherical equivalent; UDVA, uncorrected distance visual acuity; UIVA, uncorrected intermediate visual acuity; UNVA, uncorrected near visual acuity; CDVA, corrected distance visual acuity; CIVA, corrected intermediate visual acuity. 
Table 3 The correlation analysis between preoperative variables and postoperative spherical equivalent at 3 months

\begin{tabular}{lll}
\hline & $\boldsymbol{R}$ & $\boldsymbol{p}$-value \\
\hline Axial length $(\mathrm{mm})$ & -0.159 & 0.479 \\
Anterior chamber depth & -0.47 & 0.037 \\
Flattest meridian (K1) & -0.072 & 0.752 \\
Steepest meridian (K2) & -0.065 & 0.770 \\
Intraocular lens power & -0.47 & 0.037 \\
Preoperative estimation error & -0.064 & 0.768 \\
\hline
\end{tabular}

flattest meridian (K1), steepest meridian (K2), IOL power, predicted refraction and postoperative spherical equivalent at 3 months; the results are summarized in Table 3. Linear and nonlinear regression analyses were performed to establish a fit equation for adjusted Haigis formula and included those variables showing high correlation with postoperative spherical diopter.

A significant correlation was found between spherical equivalent 3 months postoperatively and preoperative ACD ( $r=-0.470, p=0.037)$. No significant correlations were observed between spherical equivalent and other preoperative variables, including IOL power $(r=-0.470$, $p=0.037), \mathrm{AL}(r=-0.159, p=0.479), \mathrm{K} 1(r=-0.072, p=0.752)$, $\mathrm{K} 2(r=-0.065, p=0.770)$ or preoperative estimation error $(r=-0.064, p=0.768)$.

As shown in Figure 1, the relationship between the preoperative $\mathrm{ACD}$ and spherical equivalent was consistent with the quadratic function curve (formula $\mathrm{Y}=14.62-8.78 \mathrm{X}+1.27 \mathrm{X}^{2}$,

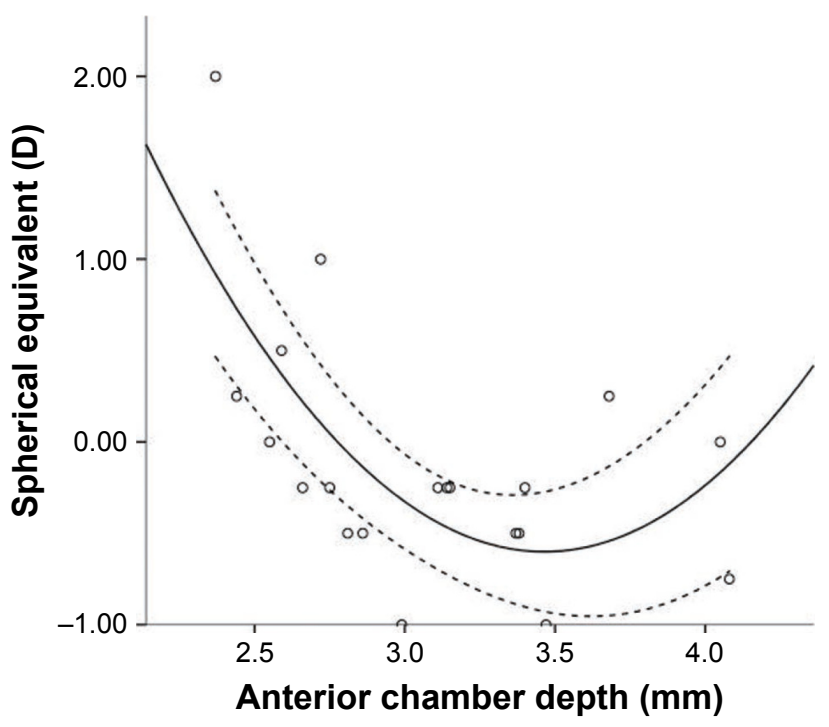

Figure I The adjusted quadratic curve for preoperative anterior chamber depth (ACD) and the spherical equivalent (SE). The $x$-axis represents SE and $y$-axis preoperative ACD. For ACD 2.8-4.12 mm, the postoperative SE was easy to shift to myopia, while for $A C D<2.8$ or $>4.12 \mathrm{~mm}$, the postoperative SE was prone to hyperopia. $p=0.007)$. When ACD was $2.8-4.12 \mathrm{~mm}$, the postoperative spherical equivalent was easily shifted to myopia. When ACD was $<2.8$ or $>4.12 \mathrm{~mm}$, the postoperative spherical equivalent trended toward hyperopia.

\section{Defocus curve}

Defocus curve depicted according to the VA at various deviations is summarized in Table 4. In this study, VA was $+0.07 \pm 0.08 \log$ MAR $(0.0 \mathrm{D}$, at ETDRS $4 \mathrm{~m}),+0.25 \pm 0.19$ $\log$ MAR $(-3.0 \mathrm{D}, 30 \mathrm{~cm})$ and $0.14 \pm 0.19 \log$ MAR $(-1.0 \mathrm{D}$, $1 \mathrm{~m})$. The defocus curve was observed to be stable, which provided continuous and acceptable vision at all distances shown in Table 2.

\section{Contrast sensitivity curve}

The contrast sensitivity in different spatial frequencies under photopic, mesopic, photopic with glare and mesopic with glare conditions at baseline and 3 months postoperatively is summarized in Table 5. The contrast sensitivity of each spatial frequency significantly improved at 1 week, 1 month and 3 months postoperatively compared to the baseline. The maximum value was reached at 1 week and maintained stable during the follow-up under photopic and photopic with glare conditions. Under mesopic with glare conditions, a significant difference was observed at 1 week, 1 month and 3 months postoperatively for medium spatial frequencies at 3 cycles/ degree $(p<0.0001)$ and $6 \mathrm{c} / \mathrm{d}(p<0.01)$; however, the results were not significant for low spatial frequency until 3 months at $1.5 \mathrm{c} / \mathrm{d}$ or high spatial frequencies during the follow-up at 12 and $18 \mathrm{c} / \mathrm{d}$. The observations under mesopic conditions

Table 4 Defocus curve and visual acuity at different deviations

\begin{tabular}{llll}
\hline $\begin{array}{l}\text { Add } \\
\text { (D) }\end{array}$ & $\begin{array}{l}\text { Distance } \\
(\mathbf{m})\end{array}$ & LogMAR & \\
\cline { 3 - 4 } & - & 0.52 & SD \\
\hline 2 & - & 0.40 & 0.20 \\
1.5 & - & 0.27 & 0.18 \\
1 & - & 0.14 & 0.16 \\
0.5 & 4 & 0.07 & 0.11 \\
0.0 & 2 & 0.06 & 0.08 \\
-0.5 & 1 & 0.14 & 0.10 \\
-1.0 & 0.67 & 0.18 & 0.19 \\
-1.5 & 0.5 & 0.23 & 0.15 \\
-2.0 & 0.4 & 0.24 & 0.17 \\
-2.5 & 0.33 & 0.25 & 0.18 \\
-3.0 & 0.29 & 0.33 & 0.19 \\
-3.5 & 0.25 & 0.43 & 0.16 \\
-4.0 & 0.22 & 0.54 & 0.18 \\
-4.5 & 0.2 & 0.68 & 0.19 \\
-5.0 & -5 & & 0.15 \\
\hline Abbrevition & & &
\end{tabular}

Abbreviation: SD, standard deviation. 
Table 5 Contrast sensitivity of different spatial frequencies under photopic, mesopic, photopic with glare and mesopic with glare preoperatively and I week, I month and 3 months postoperatively

\begin{tabular}{|c|c|c|c|c|}
\hline \multirow{2}{*}{$\begin{array}{l}\text { Spatial } \\
\text { frequency (c/d) }\end{array}$} & \multirow[t]{2}{*}{ Preoperation } & \multicolumn{3}{|c|}{ Postoperation } \\
\hline & & I week & I month & 3 months \\
\hline \multicolumn{5}{|l|}{ Mesopic } \\
\hline 1.5 & $0.68 \pm 0.42$ & $1.12 \pm 0.28 *$ & $1.17 \pm 0.30 *$ & $1.87 \pm 0.22^{*}$ \\
\hline 3 & $0.65 \pm 0.55$ & $1.32 \pm 0.34 *$ & $1.33 \pm 0.32 *$ & $1.35 \pm 0.35 *$ \\
\hline 6 & $0.19 \pm 0.45$ & $0.73 \pm 0.66 *$ & $0.75 \pm 0.68 *$ & $0.99 \pm 0.63^{*, \alpha}$ \\
\hline 12 & $0.03 \pm 0.16$ & $0.07 \pm 0.29$ & $0.18 \pm 0.42 *$ & $0.25 \pm 0.48^{* \# \#}$ \\
\hline 18 & $0.02 \pm 0.11$ & $0.07 \pm 0.24$ & $0.15 \pm 0.32 *$ & $0.10 \pm 0.30$ \\
\hline \multicolumn{5}{|l|}{ Mesopic with glare } \\
\hline 1.5 & $0.50 \pm 0.45$ & $0.72 \pm 0.54$ & $0.77 \pm 0.57$ & $0.88 \pm 0.46 *$ \\
\hline 3 & $0.36 \pm 0.53$ & $0.94 \pm 0.60^{*}$ & $1.03 \pm 0.59 *$ & $1.15 \pm 0.46^{* * \#}$ \\
\hline 6 & $0.05 \pm 0.28$ & $0.34 \pm 0.58 *$ & $0.38 \pm 0.6 I^{*}$ & $0.50 \pm 0.62 *$ \\
\hline 12 & $0.03 \pm 0.16$ & $0.04 \pm 0.22$ & $0.13 \pm 0.36$ & $0.04 \pm 0.20$ \\
\hline 18 & $0.00 \pm 0.00$ & $0.03 \pm 0.16$ & $0.03 \pm 0.15$ & $0.03 \pm 0.17$ \\
\hline \multicolumn{5}{|l|}{ Photopic } \\
\hline 1.5 & $0.82 \pm 0.38$ & $1.23 \pm 0.16 *$ & $1.24 \pm 0.19 *$ & $1.18 \pm 0.29 *$ \\
\hline 3 & $1.00 \pm 0.48$ & $1.52 \pm 0.23^{*}$ & $1.56 \pm 0.23 *$ & $1.57 \pm 0.20^{*}$ \\
\hline 6 & $0.68 \pm 0.62$ & $1.40 \pm 0.45^{*}$ & $1.39 \pm 0.45^{*}$ & $1.40 \pm 0.46^{*}$ \\
\hline 12 & $0.05 \pm 0.27$ & $0.67 \pm 0.6 I^{*}$ & $0.63 \pm 0.64 *$ & $0.72 \pm 0.64^{*}$ \\
\hline 18 & $0.04 \pm 0.22$ & $0.26 \pm 0.42 *$ & $0.26 \pm 0.45^{*}$ & $0.26 \pm 0.45^{*}$ \\
\hline \multicolumn{5}{|l|}{ Photopic with glare } \\
\hline 1.5 & $0.84 \pm 0.34$ & $1.27 \pm 0.34 *$ & $1.28 \pm 0.32 *$ & $\mid .27 \pm 0.3 \mathrm{I} *$ \\
\hline 3 & $1.00 \pm 0.53$ & $1.53 \pm 0.37^{*}$ & $1.57 \pm 0.39 *$ & $1.62 \pm 0.22 *$ \\
\hline 6 & $0.80 \pm 0.54$ & $1.18 \pm 0.69 *$ & $1.35 \pm 0.52 *$ & I. $45 \pm 0.36 *$ \\
\hline 12 & $0.07 \pm 0.29$ & $0.63 \pm 0.6 I^{*}$ & $0.70 \pm 0.61 *$ & $0.70 \pm 0.60 *$ \\
\hline 18 & $0.03 \pm 0.20$ & $0.32 \pm 0.45^{*}$ & $0.23 \pm 0.42 *$ & $0.30 \pm 0.43^{*}$ \\
\hline
\end{tabular}

Notes: ${ }^{*} p<0.05$ vs baseline; ${ }^{*} p<0.05$ vs I week; ${ }^{\alpha} p<0.05$ vs I month.

at low-to-medium spatial frequency were similar to those observed under photopic conditions, but at high spatial frequency, very small improvement was observed at 1 month.

\section{Satisfaction questionnaire}

Table 6 summarizes the results of the patients' subjective evaluation of satisfaction. At 3 months postoperatively, the questionnaire, targeting common symptoms observed in

Table 6 Results of satisfaction questionnaire

\begin{tabular}{|c|c|c|c|}
\hline & Never & Sometimes & Always \\
\hline $\begin{array}{l}\text { Discomfort performing } \\
\text { near-vision tasks }\end{array}$ & $27(100 \%)$ & 0 & 0 \\
\hline $\begin{array}{l}\text { Presence of headache if } \\
\text { reading }>15 \text { minutes }\end{array}$ & $27(100 \%)$ & 0 & 0 \\
\hline $\begin{array}{l}\text { When reading, text becomes } \\
\text { unclear }\end{array}$ & $27(100 \%)$ & 0 & 0 \\
\hline Diplopia when reading & $26(96.3 \%)$ & I (3.7\%) & 0 \\
\hline $\begin{array}{l}\text { Inability to engage in computer } \\
\text { work for prolonged periods }\end{array}$ & 27 (100\%) & 0 & 0 \\
\hline Inability to see clearly at near & 27 (I00\%) & 0 & 0 \\
\hline Need for spectacle correction & $27(100 \%)$ & 0 & 0 \\
\hline Presence of glare & $27(100 \%)$ & 0 & 0 \\
\hline Presence of halo & $27(100 \%)$ & 0 & 0 \\
\hline
\end{tabular}

presbyopia, in eyes with traditional IOLs and eyes with visual fatigue, was administered. All patients reported achieving spectacle independence. One patient (3.7\%) reported occasional diplopia during reading at month 3.

\section{Discussion}

Traditional cataract surgery with single-focus IOL implantation can provide good VA outcomes for patients; however, many still require spectacle correction postoperatively. ${ }^{16}$ Currently, multifocal IOLs have been developed and adopted with the intention of increasing the depth of field at varying distances. ${ }^{7,17}$ The most commonly applied optical designs are refraction, diffraction and a combination of both. The AT LISA tri 839MP is a single-piece, diffractive, triple-focus preloaded IOL, with a $6.0 \mathrm{~mm}$ biconvex lens and a total length of $11.0 \mathrm{~mm} .{ }^{9}$ As a foldable hydrophilic acrylic IOL, its water content is $25 \%$ and it has a hydrophobic surface. The area of the optical zone is divided into a main zone and phase zones. The main zone is a trifocal region with a diameter of $4.34 \mathrm{~mm}$. The phase zone is the bifocal peripheral area from 4.34 to $6.00 \mathrm{~mm}$. The additional correction for near vision is $+3.33 \mathrm{D}$ and for intermediate vision is $+1.66 \mathrm{D}$. The lens 
design allocates $50 \%$ of the light-to-distance vision, $20 \%$ to intermediate and $30 \%$ for near vision with an $85.7 \%$ overall transmittance. There is a slow transition area between the main zone and phase zones, referred to as a diffraction structure, used to reduce the disturbing light phenomena including glare and halo and improve the overall quality of retinal imaging and $\mathrm{VA}$.

In the present study, 26 patients with age-related cataract were enrolled and evaluated for distance, intermediate and near VA before and after implantation of AT LISA tri 839MP trifocal IOL. UDVA, UIVA and UNVA were 0.3 (logMAR) or better in $100 \%, 83.3 \%$ and $87.5 \%$ of eyes, respectively, 3 months after surgery. This result indicates that the vast majority of patients achieved the ideal uncorrected postoperative VA. The results were similar to other research about AT LISA tri 839MP IOL implantation. Mendicute et al reported that $94.7 \%$ (195 eyes), $87.5 \%$ (176 eyes) and $92.5 \%$ (160 eyes) had UDVA, UIVA and UNVA of $0.3 \log$ MAR or better, respectively. ${ }^{18}$ More specifically, UDVA was $0.05 \pm 0.10 \log$ MAR, UIVA was $0.23 \pm 0.12 \log$ MAR and UNVA was $0.21 \pm 0.15 \log \mathrm{MAR}$, at 3 months postoperatively. These results are similar to those reported by Mojzis et al in their study of 60 eyes, 3 months following implantation of the AT LISA tri 839MP IOL. They reported UDVA of $0.03 \pm 0.09 \log$ MAR, UIVA of $0.24 \pm 0.10 \log$ MAR and UNVA of $0.36 \pm 0.12 \log$ MAR. ${ }^{9}$

In the current study, the observed improvements in VA remained stable during the follow-up period. There were no significant changes in UIVA or UNVA, or CDVA, CIVA or CNVA between the 1-week and 3-month time points. There was an additional improvement in UDVA at 3 months, which may be associated with early anterior chamber inflammation that can affect distance vision. Additional studies evaluating other trifocal IOLs have also shown good distance VA; however, other studies evaluating multifocal IOLs have demonstrated improvement in intermediate VA, while near or distance VA was somewhat sacrificed. ${ }^{19-26}$ Considering the earlier studies and the present findings, the AT LISA tri 839MP trifocal IOL provided sustainable improvement of $\mathrm{VA}$ at near, intermediate and distance.

Further correlation analysis between preoperative variables and postoperative spherical diopter was performed and regression analyses were subsequently conducted to evaluate the adjusted Haigis formula. Notably, the 3-month spherical equivalent and preoperative ACD were negatively correlated, and there was no correlation with the preoperative estimation error. Further analysis for postoperative spherical equivalent and preoperative ACD demonstrated the following regressive formula: $\mathrm{Y}=14.62-8.78 \mathrm{X}+1.27 \mathrm{X}^{2}$. It is well known that the parameters related to the estimation error include corneal curvature, ACD and AL in commonly used formulae to predict postoperative spherical equivalent. ${ }^{27,28}$ Several studies state that postoperative refractive error is correlated with ACD and highlight the significance of the ACD; however, there appears to be paucity of a clear statement regarding the proposed correction formula or use of an additional formula to amend the postoperative refractive error. ${ }^{29,30}$ In the present study, the Haigis formula was further optimized according to various classifications of preoperative ACD. When the preoperative ACD was $<2.8$ or $>4.12 \mathrm{~mm}$, the effective lens position (ELP) predicted was smaller than the actual value according to the Haigis formula, resulting in a hyperopic shift. When the ACD was $2.8-4.12 \mathrm{~mm}$, the Haigis formula predicted an IOL ELP larger than the actual value, resulting in a myopic shift. Thus, applying the Haigis formula to calculate the IOL power targeted for emmetropia, the preoperative IOL power should be near -0.5 and $-0.75 \mathrm{D}$ for $\mathrm{ACD}<2.55$ and $>4.37 \mathrm{~mm}$, respectively, while ACD between 3.25 and $3.66 \mathrm{~mm}$ should be close to $0.5 \mathrm{D}$. Applying the second correction can improve the accuracy of the IOL measurement.

To further investigate VA following IOL implantation, we conducted defocus curve assessment before and after surgery. The defocus curve can demonstrate patients' refractive error at varying distances. The bimodal defocus curve of the classic bifocal IOL is obvious, especially for AcrysofRestor C3.0 D, AcrysofRestor C4.0 D IOLs (Alcon Laboratories, Inc.), Tecnis ZM 900 multifocal IOL (Abbott Medical Optics, Inc.) and Acri.Lisa 366 D IOL (Carl Zeiss Meditec, AG). The defocus curves of these bifocal IOLs demonstrate a significant downward trend at -1.5 D. ${ }^{18,31-33}$ In our study, after implantation of AT LISA tri 839MP IOL, the mean VA was $0.14 \pm 0.11 \log$ MAR when the defocus curve value was $+0.5 \mathrm{D}$, and $0.18 \pm 0.15 \log \mathrm{MAR}$ when the defocus curve value was $-1.5 \mathrm{D}$. There was no significant difference between them, and the defocus curve remained relatively stable ranging from -1.5 to $0.5 \mathrm{D}$ (within a visual distance of $67 \mathrm{~cm}$ to $4 \mathrm{~m}$ ). At these distances, the mean change in VA was $0.12 \log$ MAR or better $(0.18-0.06$ $\log$ MAR), a result expected with the optic design of the AT LISA tri 839MP IOL. The lens design emphasizes an increase to the focus at intermediate distances, so that the patients obtain an acceptable intermediate VA. ${ }^{9}$ In the present study, the change in the defocus curve was continuous and slow, indicating that patients' intermediate VA was more comfortable and durable. 
Contrast sensitivity represents the reciprocal of the threshold contrast of the human eye at different spatial frequencies. ${ }^{34}$ In our study, under photopic, mesopic, photopic with glare and mesopic with glare conditions, contrast sensitivity in the various spatial frequencies improved at 3 months, when compared to baseline. However, under mesopic with glare, the contrast sensitivity curve of middle and high spatial frequencies decreased rapidly and did not change significantly compared to preoperative assessment, indicating that glare had impacted the quality of retinal imaging after pupil dilation. This result is consistent with the analysis of AT LISA tri 839MP aberration published by Mojzis et al. ${ }^{35}$ Overall, postoperative visual function improved and remained stable following AT LISA tri 839MP IOL implantation under photopic and mesopic conditions.

Although not a validated questionnaire, results of the satisfaction survey indicated that at 3 months, only one patient occasionally experienced glare. No other adverse circumstances or dissatisfaction was reported by patients in this study. The patient who reported the glare had a preoperative VA of $0.9 \log$ MAR, with a flat corneal curvature. The K1 and K2 were 41.21 and 41.87, respectively, and the ACD was shallow $(2.37 \mathrm{~mm})$. The AL was normal at $23.75 \mathrm{~mm}$. Considering the postoperative spherical equivalent of $+2.00 \mathrm{D}$, the less-than-optimal result was likely due to the IOL power selection in this patient. Preoperative assessment should be comprehensive, and multiple factors should be carefully considered when selecting IOL power. At 3 months postoperatively, the cohort reported a spectacle independence rate of $100 \%$, and absence of glare phenomenon. The postoperative satisfaction in the present study is similar to the data provided by other studies. ${ }^{18,36}$

\section{Conclusion}

The results of the present study demonstrate that implantation of the trifocal design AT LISA tri 839MP IOL is capable of providing patients with cataract with safe, stable and ( $\mathrm{mm}$ ) VA improvement at variable distances and light frequencies, as well as high postoperative satisfaction. As well, the use of preoperative ACD is effective in optimizing the Haigis formula to target and predict postoperative refraction. We do acknowledge the limitations to the study, including the small number of patients, and limited data available to adequately fit the curve and make statistical conclusions regarding the corrected formula.

\section{Acknowledgment}

Yang Yang and Huibin Lv are co-first authors.

\section{Disclosure}

The authors report no conflicts of interest in this work. None of the authors have any financial or proprietary interest in any of the material or methods mentioned and there are no funding sources to report.

\section{References}

1. Lee CM, Afshari NA. The global state of cataract blindness. Curr Opin Ophthalmol. 2017;28(1):98-103.

2. Mundy KM, Nichols E, Lindsey J. Socioeconomic disparities in cataract prevalence, characteristics, and management. Semin Ophthalmol. 2016;31(4):358-363.

3. Wang SY, Stem MS, Oren G, et al. Patient-centered and visual quality outcomes of premium cataract surgery: a systematic review. Eur $J$ Ophthalmol. 2017;27(4):387-401.

4. Hahn U, Krummenauer F. Results and methodology of cost-utility evaluation of cataract surgery in developed countries: quality-adjusted life years and cataract. $J$ Cataract Refract Surg. 2017;43(6):839-847.

5. de Silva SR, Evans JR, Kirthi V, et al. Multifocal versus monofocal intraocular lenses after cataract extraction. Cochrane Database Syst Rev. 2016;12:CD003169.

6. Calladine D, Evans JR, Shah S, et al. Multifocal versus monofocal intraocular lenses after cataract extraction. Cochrane Database Syst Rev. 2012;(9):CD003169.

7. Leyland M, Zinicola E. Multifocal versus monofocal intraocular lenses in cataract surgery: a systematic review. Ophthalmology. 2003; 110(9):1789-1798.

8. Rojas MJ, Yeu E. An update on new low add multifocal intraocular lenses. Curr Opin Ophthalmol. 2016;27(4):292-297.

9. Mojzis P, Majerova K, Hrckova L, Pinero DP. Implantation of a diffractive trifocal intraocular lens: one-year follow-up. J Cataract Refract Surg. 2015;41(8):1623-1630.

10. Kretz FT, Breyer D, Diakonis VF, et al. Clinical outcomes after binocular implantation of a new trifocal diffractive intraocular lens. J Ophthalmol. 2015;2015:962891.

11. Voskresenskaya A, Pozdeyeva N, Pashtaev N, et al. Initial results of trifocal diffractive IOL implantation. Graefes Arch Clin Exp Ophthalmol. 2010;248(9):1299-1306.

12. Gatinel D, Pagnoulle C, Houbrechts Y, Gobin L. Design and qualification of a diffractive trifocal optical profile for intraocular lenses. J Cataract Refract Surg. 2011;37(11):2060-2067.

13. Alfonso JF, Fernandez-Vega Cueto L, Belda-Salmeron L, et al. Visual function after implantation of a diffractive aspheric trifocal intraocular lens. Eur J Ophthalmol. 2016;26(5):405-411.

14. Reitblat O, Levy A, Kleinmann G, et al. Intraocular lens power calculation for eyes with high and low average keratometry readings: comparison between various formulas. J Cataract Refract Surg. 2017; 43(9):1149-1156.

15. Melles RB, Holladay JT, Chang WJ. Accuracy of intraocular lens calculation formulas. Ophthalmology. 2018;125(2):169-178.

16. Ong HS, Evans JR, Allan BD. Accommodative intraocular lens versus standard monofocal intraocular lens implantation in cataract surgery. Cochrane Database Syst Rev. 2014;(5):CD009667.

17. Menapace R. Developments in modern cataract surgery - a critical overview. Ther Umsch. 2016;73(2):53-59.

18. Mendicute J, Kapp A, Levy P, et al. Evaluation of visual outcomes and patient satisfaction after implantation of a diffractive trifocal intraocular lens. J Cataract Refract Surg. 2016;42(2):203-210.

19. Kohnen T, Nuijts R, Levy P, et al. Visual function after bilateral implantation of apodized diffractive aspheric multifocal intraocular lenses with a +3.0 D addition. J Cataract Refract Surg. 2009;35(12):2062-2069.

20. Cochener B, Vryghem J, Rozot P, et al. Visual and refractive outcomes after implantation of a fully diffractive trifocal lens. Clin Ophthalmol. 2012;6:1421-1427. 
21. Marques JP, Rosa AM, Quendera B, et al. Quantitative evaluation of visual function 12 months after bilateral implantation of a diffractive trifocal IOL. Eur J Ophthalmol. 2015;25(6):516-524.

22. Sheppard AL, Shah S, Bhatt U, et al. Visual outcomes and subjective experience after bilateral implantation of a new diffractive trifocal intraocular lens. J Cataract Refract Surg. 2013;39(3):343-349.

23. Vryghem JC, Heireman S. Visual performance after the implantation of a new trifocal intraocular lens. Clin Ophthalmol. 2013;7:1957-1965.

24. Gatinel D, Houbrechts Y. Comparison of bifocal and trifocal diffractive and refractive intraocular lenses using an optical bench. J Cataract Refract Surg. 2013;39(7):1093-1099.

25. Alfonso JF, Fernandez-Vega L, Puchades C, Montes-Mico R. Intermediate visual function with different multifocal intraocular lens models. J Cataract Refract Surg. 2010;36(5):733-739.

26. Ferreira TB, Marques EF, Rodrigues A, Montes-Mico R. Visual and optical outcomes of a diffractive multifocal toric intraocular lens. J Cataract Refract Surg. 2013;39(7):1029-1035.

27. Savini G, Hoffer KJ, Lomoriello DS, Ducoli P. Simulated keratometry versus total corneal power by ray tracing: a comparison in prediction accuracy of intraocular lens power. Cornea. 2017;36(11):1368-1372.

28. Zhang Z, Thomas LW, Leu SY, Carter S, Garg S. Refractive outcomes of intraoperative wavefront aberrometry versus optical biometry alone for intraocular lens power calculation. Indian J Ophthalmol. 2017; 65(9):813-817.

29. Jeong J, Song H, Lee JK, et al. The effect of ocular biometric factors on the accuracy of various IOL power calculation formulas. $B M C$ Ophthalmol. 2017;17(1):62.
30. Sedaghat MR, Azimi A, Arasteh P, et al. The relationship between anterior chamber depth, axial length and intraocular lens power among candidates for cataract surgery. Electron Physician. 2016;8(10): 3127-3131.

31. Carballo-Alvarez J, Vazquez-Molini JM, Sanz-Fernandez JC, et al. Visual outcomes after bilateral trifocal diffractive intraocular lens implantation. BMC Ophthalmol. 2015;15:26.

32. Moyal L, Abrieu-Lacaille M, Bonnel S, et al. Comparison of two different surgical treatments of presbyopia for hyperopic patients over 55 years old: Presbylasik (Supracor) and Prelex (presbyopic lens exchange). J Fr Ophtalmol. 2015;38(4):306-315.

33. Carson D, Hill WE, Hong X, Karakelle M. Optical bench performance of AcrySof(®) IQ ReSTOR(®), AT LISA(®) tri, and FineVision(®) intraocular lenses. Clin Ophthalmol. 2014;8:2105-2113.

34. Richman J, Spaeth GL, Wirostko B. Contrast sensitivity basics and a critique of currently available tests. J Cataract Refract Surg. 2013;39(7): 1100-1106.

35. Mojzis P, Pena-Garcia P, Liehneova I, et al. Outcomes of a new diffractive trifocal intraocular lens. J Cataract Refract Surg. 2014;40(1): 60-69.

36. Kretz FT, Choi CY, Muller M, et al. Visual outcomes, patient satisfaction and spectacle independence with a trifocal diffractive intraocular lens. Korean J Ophthalmol. 2016;30(3):180-191.
Clinical Ophthalmology

\section{Publish your work in this journal}

Clinical Ophthalmology is an international, peer-reviewed journal covering all subspecialties within ophthalmology. Key topics include: Optometry; Visual science; Pharmacology and drug therapy in eye diseases; Basic Sciences; Primary and Secondary eye care; Patient Safety and Quality of Care Improvements. This journal is indexed on

\section{Dovepress}

PubMed Central and CAS, and is the official journal of The Society of Clinical Ophthalmology (SCO). The manuscript management system is completely online and includes a very quick and fair peer-review system, which is all easy to use. Visit http://www.dovepress.com/ testimonials.php to read real quotes from published authors. 